\title{
СОВРЕМЕННЫЕ ИССЛЕДОВАНИЯ ВЛИЯНИЯ ТЕТРАГИДРОКАННАБИНОЛА НА ВЫСШИЕ ПСИХИЧЕСКИЕ ФУНКЦИИ
}

\author{
Е.В. ЛАРИОНОВА ${ }^{\mathrm{a}, \mathrm{b}}$, А.А. ШУВАЛОВА
}

\begin{abstract}
${ }^{a}$ Институт высшей нервной деятельности и нейрофизиологии Российской академии наук, 117485, Россия, Москва, ул. Бутлерова, д. 5 а

${ }^{b}$ Московский научно-практический центр наркологии, 109390, Россия, Москва, ул. Люблинская, d. $37 / 1$
\end{abstract}

\section{Резюме}

Употребление каннабиноидов становится все более распространенным в современном мире, с этим связан рост научного интереса к проблеме последствий употребления этого наркотика для человеческого организма, главным образом для центральной нервной системы. В данном обзоре мы рассмотрим исследования, затрагивающие два основных вопроса: каковы эти последствия и насколько они устойчивы? В России проблеме употребления каннабиноидов по сравнению с другими наркотическими веществами уделяется крайне мало внимания: существуют единичные работы, затрагивающие эту тему. В зарубежных исследованиях в основном показаны нарушения исполнительных функций и рабочей памяти. Однако подход, используемый зарубежными исследователями, не созвучен отечественному нейропсихологическому факторному анализу - качественной оценке состояния высших психических функций. Более того, основная часть зарубежных исследований направлена на изучение острых эффектов каннабиноидов, т.е. эффектов, возникающих непосредственно после употребления наркотика, в то время как вопросы об отставленных эффектах и обратимости негативных последствий употребления остаются без ответа. Тем не менее результаты немногочисленных работ, касающихся отставленных эффектов употребления наркотика, несмотря на противоречивые данные, позволяют говорить о том, что нарушения высших психических функций сохраняются, по крайней мере, в течение месяца после последнего приема наркотика. На наш взгляд, использование синдромного анализа для изучения возможных нарушений высших психических функций при употреблении каннабиноидов позволило бы наиболее эффективно подойти к решению этой проблемы и ответить на вопрос, насколько устойчивы эти нарушения.

Ключевые слова: высшие психические функции, каннабиноиды, исполнительные функции, память, тетрагидроканнабинол. 


\section{Введение}

За последние несколько лет увеличилось количество исследований, посвященных влиянию каннабиноидов на высшие психические функции. Приводятся противоречивые данные относительно того, какие именно последствия влечет за собой употребление этого наркотика и насколько эти последствия устойчивы. Нарушения могут проявляться на различных уровнях, начиная с простых, таких как координация движений, и заканчивая принятием решений, планированием и контролем. В данном обзоре мы рассмотрим основные результаты работ, связанных с этой проблематикой, и попытаемся оценить их с позиции отечественной нейропсихологии.

\section{Влияние каннабиноидов на исполнительные функции}

Пристальному изучению были подвержены исполнительные функции - совокупность процессов, связанных с планированием и контролем текущей деятельности. Центральный симптом зависимости - желание употребить наркотик снова независимо от последствий, таким образом, исполнительные функции являются напрямую связанными с формированием зависимости (Hester et al., 2010). Их нарушение может негативно сказаться и на приверженности лечению, увеличивая возможность рецидива. Таким образом, затрагивается не только аспект выявления нарушений, но и восстановления этих функций для ремиссии таких пациентов.

При исследовании острых эффектов каннабиноидов (испытуемые употребляют наркотик перед началом эксперимента) обнаруживаются нарушения когнитивного контроля, связанного с импульсивностью (McDonald et al., 2003; Ranganathan, D'Souza, 2006), и планирования (Ranganathan, D’Souza, 2006). Дж. Макдональд c coaвт. (McDonald et al., 2003) определили импульсивное поведение как неспособность подавлять неадекватные действия, нечувствительность к последствиям, искаженное восприятие времени и персеверации поведения. Использовалось несколько тестов, связанных с различными аспектами импульсивного поведения под воздействием марихуаны; были выявлены нарушения при выполнении лишь одного из них. Авторы делают выводы, что тетрагидроканнабинол (ТГК), основной психоактивный компонент конопли, влияет на сферу импульсивности и саморегуляции.

Оценивались (Ranganathan, D’Souza, 2006) острые эффекты у куривших наркотик в среднем три раза в месяц в течение последних четырех лет. Авторы приходят к выводу, что существует доза-зависимый эффект в отношении когнитивного контроля, нарушение которого наблюдалось при более высоких дозах. Важно отметить, однако, что обследованные достаточно редко употребляли наркотик и могли быть более чувствительны к острым эффектам марихуаны по сравнению с хроническими курильщиками. Последние не показали нарушений в исследовании, посвященном различным аспектам исполнительных функций, таких как способность к абстрагированию, гибкость мышления, контроль (Hart, van Gorp, 2001). Эти испытуемые употребляли наркотик в среднем в течение четырех лет. Было проведено 
три сессии с различной концентрацией ТГК $(0 \%, 1.8 \%$ и 3.9\%) - между группами не было выявлено достоверных различий. По мнению авторов, возможное объяснение состоит в том, что у хронических курильщиков в конечном итоге развиваются компенсаторные стратегии, и они склонны быть более осторожны в выполнении задач после употребления.

\section{Исследование мнестической деятельности}

Работы, посвященные исследованию мнестической деятельности, также выявляют ряд нарушений, возникающих под воздействием наркотика. Своеобразие зарубежного подхода к изучению памяти, в отличие от традиционных отечественных нейропсихологических исследований, заключается в том, что зарубежные исследователи занимаются таким явлением, как рабочая память (working memory), включающим в себя функции непосредственной памяти и внимания. Так, в исследовании М. Боссонга с соавт. (Bossong et al., 2012) с использованием парадигмы Стернберга было показано значимое снижение производительности рабочей памяти после воздействия ТГК, также у испытуемых после воздействия ТГК при низкой нагрузке наблюдалась повышенная активность некоторых областей мозга, связанных с функциями рабочей памяти, по сравнению с контрольной группой, у которой производительность была лучше независимо от нагрузки, а мозговая активность увеличивалась с ростом нагрузки. К. Бёкер с соавт., исследуя острые эффекты, получили схожие резуль- таты: он проводил тестирование памяти через час после курения сигарет, содержащих ТГК, используя ряды чисел от 2 до 5, затем числозонд, и испытуемый должен был ответить, относится ли «зонд» к предъявлявшемуся числовому ряду. Было показано снижение продуктивности рабочей памяти при приеме наркотика (Böcker et al., 2010).

\section{Высшие психические функции и многократное употребление каннабиноидов}

При исследовании эффектов, не связанных с субъективно ощущаемым наркотическим опьянением, часть работ выполнена при воздержании испытуемых от употребления наркотиков в течение небольшого периода времени (несколько часов), другая - более длительного (несколько дней, месяцев).

В 1985 г. было проведено исследование (Barnett et al., 1985), в процессе которого было отчетливо продемонстрировано, что даже «опытные» потребители марихуаны после выкуривания одной сигареты существенно хуже выполняли на тренажерах сложные операторские задачи (отслеживание параллельно двигающегося автомобиля или разделение внимания между несколькими объектами на дороге), чем некурящие люди и курильщики табака. Причем нарушение этих функций продолжается в течение 8 часов - гораздо дольше, чем субъективно ощущаемое наркотическое опьянение.

Наиболее часто используемыми нейропсихологическими методиками в подобных работах являются тест Струпа и Висконсинский тест 
сортировки карточек (Wisconsin Card Sorting Test, WCST), первый связан, прежде всего, с когнитивным контролем, второй - с планированием, регуляцией и контролем, гибкостью мышления, способностью к переключению внимания.

Так, в одном из исследований (Solowij et al., 2002) с применением этих тестов, посвященном хроническому употреблению марихуаны и влиянию продолжительности ее употребления на когнитивные функции, сравнивались две группы употреблявших наркотик, различающиеся по длительности стажа (около 10 и около 20 лет употребления) и воздерживающиеся от курения 17 часов, и контрольная группа, состоящая из испытуемых, не употреблявших каннабис. Достоверные различия были выявлены только в WCST, причем максимальное количество ошибок наблюдалось у испытуемых с большим стажем употребления. Похожие результаты были получены в исследовании (Рope et al., 1996), в котором после 19 часов воздержания более выраженные нарушения наблюдались у испытуемых, которые чаще употребляли наркотик в прошлом: в WCST у них наблюдалось больше ошибок по типу персевераций.

У испытуемых другого исследования (Pope, Yurgelun-Todd, 2001) срок воздержания достигал 28 дней, они различались по стажу употребления и тестировались на 0, 1, 7 и 28-й дни. Использовались тест Струпа и WCST. Никаких существенных различий не было обнаружено между группами на 28-й день. Авторы пришли к выводу, что, по крайней мере, некоторые нарушения являются обратимыми. В схожем исследова- нии (Bolla et al., 2002) на 28-й день воздержания нарушения у испытуемых с большим стажем употребления сохранялись: они имели больше нарушений в WCST. В работе 2004 г. (Lyons et al., 2004) срок воздержания достигал года, а стаж употребления составлял 20 лет: в тесте Струпа и WCST достоверных различий с контрольной группой выявлено не было.

\section{Изучение острых и отсроченных эффектов каннабиноидов}

Большинство исследований посвящено непосредственным эффектам наркотика, возникающим во время или сразу после его употребления. Это, вероятно, обусловлено тем, что непосредственные эффекты легче фиксировать в экспериментах, они ярче представлены. Так, например, в отечественной литературе существуют упоминания о воздействии марихуаны на сферу восприятия (Личко, 1991), на оценку расстояния и времени (Шабанов, 2002). В целом в отечественных изданиях клиникопсихологического профиля проблема последствий употребления каннабиноидов освещена недостаточно.

Нарушения высших психических функций были показаны в целом ряде работ, посвященных изучению острых эффектов наркотика: нарушения исполнительных функций, функций внимания и памяти. Нарушения рабочей памяти сохраняются в течение нескольких недель, согласно результатам некоторых исследований (Medina et al., 2007), но, похоже, они исчезают при воздержании от наркотика (Thames et al., 2014). При изучении функции внимания испытуемые, воздерживающиеся от наркотика, пока- 
зывали меньше нарушений этой функции при увеличении периода воздержания от наркотика (Bosker et al., 2013). Существует мало исследований оценки восстановления исполнительных функций при воздержании от наркотика, хотя проведение подобных исследований важно для оптимизации программ лечения каннабиноидной зависимости (Broyd et al., 2016). Так, в исследовании С.P. Хупера с соавт. (Hooper et al., 2014) нарушения исполнительных функций у подростков были связаны с рецидивом употребления каннабиноидов в течение одного года наблюдения.

Многие работы выявляют значительные нарушения при небольших сроках воздержания, но не выявляют нарушений при длительном воздержании от употребления наркотика. Существуют различные факторы, осложняющие получение достоверных результатов в подобного рода исследованиях: необходимость учитывать сроки воздержания и частоту употребления в прошлом, употребление других наркотических веществ наряду с каннабиноидами, преморбидные особенности, наличие психиатрических и неврологических заболеваний, размеры выборки, тип используемых тестов. Одним из таких факторов является отсутствие данных о преморбидных особенностях исследуемой группы, в частности об особенностях когнитивных функций до начала употребления каннабиноидов. Так, можно предположить, что полученные различия могут быть связаны с ними и не являются последствиями употребления наркотика. Тем не менее в лонгитюдном исследовании (Meier et al., 2012) показано, что снижение когнитивных функций опреде- лялось употреблением каннабиноидов. Таким образом, проведение лонгитюдных исследований помогает преодолеть эти ограничения, однако лонгитюдные исследования сами по себе дольно трудоемкие.

В исследованиях острых эффектов каннабиноидов испытуемыми часто становятся те, кто ранее употреблял наркотик, поэтому, вероятно, остаточные эффекты ТГК могут также оказывать влияние на результаты и смешиваться с острыми эффектами. Но в отличие от исследования долговременных эффектов при исследовании острых есть возможность контролировать такие факторы, как, например, доза наркотика.

В исследованиях долговременных последствий, помимо отсутствия сведений о преморбидных особенностях когнитивных функций, такие факторы, как продолжительность, частота употребления и доза употребляемого наркотика, оказываются практически неконтролируемыми. Во-первых, подобные сведения возможно оценивать лишь со слов испытуемых, а они, как правило, затрудняются с ответами на вопросы о начале употребления наркотика, его продолжительности и частоте. Во-вторых, оценивать дозы фактически невозможно, так как испытуемые употребляют марихуану и/или гашиш, в которых концентрация дельта-9-тетрагидроканнабинола (ТГК), основного психоактивного компонента, различна.

Таким образом, необходим тщательный отбор испытуемых с проработанными критериями исключения для формирования однородной группы. Так, в некоторых исследованиях (Hart, van Gorp, 2001; Ranganathan, D'Souza, 2006) разница в возрасте испытуемых 
достигает 10 лет, в то время как в другом исследовании (McDonald et al., 2003) - 27 лет. Следует обратить внимание и на стаж употребления: более выраженные нарушения при изучении острых эффектов выявляются у испытуемых, редко употреблявших каннабиноиды. Нужно учитывать количество, частоту и продолжительность употребления наркотиков, так как эти аспекты связаны с развитием толерантности к острым эффектам.

Испытуемые большинства проведенных исследований долговременных последствий отличаются не очень длительным периодом воздержания от наркотика, что оставляет неясным ответ на вопрос об устойчивости выявленных нарушений. Несмотря на противоречивые результаты, устойчивые нарушения, по-видимому, сохраняются, по крайней мере, в течение 28 дней воздержания. Поэтому остается вопрос о влиянии хронического употребления марихуаны и обратимости этих нарушений.

\section{Сравнение зарубежного и отечественного подходов в исследованиях влияния тетрагидроканнабинола на высшие психические функции}

Нейропсихологические методы являются довольно распространенными при исследовании когнитивных нарушений. Полное обследование высших психических функций двух пациентов с диагностированной каннабиноидной зависимостью было проведено с использованием батареи классических нейропсихологических методик (Цветков, 2012). В ходе проведенного эксперимента были зафиксированы явления экстериоризации психических процессов, которые имеют место при относительно небольшом сроке злоупотребления (3 года) и были квалифицированы автором как компенсаторные симптомы. У пациента со стажем наркотизации 15 лет наблюдались выраженные нарушения нейродинамических компонентов, мышления и памяти.

В 2014 г. (Брюн и др., 2014) были описаны особенности системных связей в познавательной сфере лиц, употребляющих каннабиноиды, по сравнению с группой нормы. Главной из этих особенностей является снижение числа межфункциональных связей процессов мышления, внимания, памяти и произвольной регуляции деятельности, что интерпретируется авторами исследования как «расщепление» познавательной сферы, характерное для расстройств шизофренического спектра.

Зарубежные исследователи также применяют нейропсихологические методы для изучения последствий употребления каннабиноидов. Однако стоит учитывать, что зарубежный подход отличается от отечественного, а основные работы, связанные с оценкой последствий употребления каннабиноидов, были выполнены именно за рубежом, что накладывает отпечаток на полученные результаты и качество проводимых исследований. Вместо синдромного анализа, направленного на целый ряд функций и позволяющего выявлять как первичные, так и связанные с ними вторичные нарушения, зарубежные авторы часто ограничивались применением одной-двух однонаправленных методик, а оценка носила количественный, но не качественный характер (например: Böcker et al., 
2010; Bossong et al., 2012). В рамках отечественного нейропсихологического подхода (Лурия, 1962) данные о внешних проявлениях нарушенных функций всегда рассматриваются не с описательной точки зрения, в виде неких симптомов, а с позиции качественного анализа, ориентированного на выявление нарушенного звена, общего для различных функций и симптомов, - это позволяет подвергать полученные данные более глубокому анализу, извлекать новые сведения о возможных механизмах отправления той или иной психической функции, высказывать предположения об особенностях психического функционирования, наблюдаемых в эксперименте, таким образом, применение нейропсихологического анализа дает новые возможности при формулировании выводов.

Несомненным достоинством зарубежных исследований является широкое использование методов нейровизуализации при изучении проблемы влияния ТГК на когнитивные функции (Hell et al., 2011; Battistella et al., 2013; Ramaekers et al., 2006). Так, например, в исследовании X. Поупа с coaвt. (Pope, Yurgelun-Todd, 2001) c использованием нейропсихологических методик при воздержании от наркотика различий с группой контроля показано не было, хотя авторы высказывают мнение, что данные методы не позволили выявить менее выраженные дисфункции. Для решения этой проблемы перспективным является подход, в котором результаты нейропсихологических методик сопоставляются с данными нейровизуализации. Так, в работе Г. Ягер и P. Кан (Jager, Kahn, 2006) у группы наркотизирующихся лиц после 1 недели воздержания, по результатам функциональной магнитно-резонансной томографии (фМРТ), при выполнении заданий на запоминание и внимание зафиксированная активность мозга в левой верхнетеменной области значительно отличалась от активности в этих областях у контрольной группы. Однако исследователи не обнаружили внешних симптомов нарушений так называемой вербальной рабочей памяти (verbal working memory) и селективного внимания, в то время как в другом исследовании (Day et al., 2013) было показано нарушение рабочей памяти: испытуемыекурильщики марихуаны показали снижение производительности, увеличение времени выполнения заданий в Trail-Making Testand Digit Span. Сходные результаты были получены при изучении внимания другой группой исследователей (Eldreth et al., 2004): при воздержании от наркотика в течение нескольких недель у испытуемых, употреблявших каннабиноиды, наблюдалось снижение активности в нижнем слое передней части поясной извилины левого полушария и латеральной префронтальной коре левого полушария, а также повышение активности гиппокампа по сравнению с контрольной группой, однако психологические тесты на внимание (тест Струпа) не выявили отличий от группы контроля.

Одной из наиболее чувствительных областей к воздействию каннабиноидов является дорсолатеральная префронтальная кора, в которой сосредоточено наибольшее количество каннабиноидных рецепторов, соответственно воздействие каннабиноидов приводит к нарушению целого ряда когнитивных функций, связан- 
ных с этой областью: исполнительных функций, внимания, памяти (Eggan et al., 2010). Тем не менее эти нарушения, как показано в исследованиях долговременных эффектов каннабиноидов, не всегда выходят на поведенческий уровень, однако использование методов нейровизуализации помогает выявить эти нарушения.

Тем не менее зарубежные исследования, даже с использованием современных методов нейровизуализации, как правило, направлены на изучение отдельно взятой функции или какого-либо ее аспекта (например, вербальная память) и не оценивают всех психических функций. На наш взгляд, обоснованным является проведение комплексного нейропсихологического обследования в русле отечественного подхода с возможностью качественного анализа, попыткой проанализировать комплекс нарушенных и сохранных звеньев, а также с выявлением первичных и сопутствующих дефектов.

\section{Заключение}

Проведение исследований влияния ТГК на высшие психические функции является актуальным в свете тенденции легализации этого наркотика в мире. Особенно важным является вопрос последствий употребления каннабиноидов для когнитивных функций. Стоит отметить, что при достаточном количестве исследований, посвященных непосредственному влиянию ТГК, мало внимания уделено психологическим и когнитивным последствиям употребления этого наркотика, отставленным во времени, наблюдаемым в период воздержания от употребления. На наш взгляд, целесообразным было бы проведение комплексного нейропсихологического обследования у лиц, находящихся в состоянии воздержания в течение более длительного времени (от нескольких месяцев), дополненного данными методов нейровизуализации.

\section{Литература}

Брюн, Е. А., Шувалова, А. А., Цветков, А. В. (2014). Особенности психических функций у лиц, употребляющих каннабиноиды. Теория и практика общественного развития, 19. Режим доступа: http://teoria-practica.ru/rus/files/arhiv_zhurnala/2014/19/psychology/bryun-tsvetkov-shuvalova.pdf

Личко, А. Е. (1991). Подростковая наркология: руководство для врачей. Л.: Медицина.

Лурия, А. Р. (1962). Высшие корковые функции человека и их нарушение при локальных поражениях мозга. М.: Изд-во Московского университета.

Цветков, А. В. (2012). Особенности психической деятельности лиц, употребляющих каннабиноиды. В кн. V съезд Общероссийской общественно организащии «Российское психологическое общество». Москва, 14-18 февраля 2012 года. Научные материаль (т. 3, с. 72-73). М.: Российское психологическое общество. Режим доступа: http://www.psyrus.ru/periodicals/ pdf/v_tom_3.pdf

Шабанов, П. Д. (2002). Основы наркологии. СПб.: Лань. 
Ларионова Екатерина Владимировна - младший научный сотрудник, Институт высшей нервной деятельности и нейрофизиологии Российской академии наук; клинический психолог, Московский научно-практический центр наркологии.

Сфера научных интересов: нейропсихология, психофизиология, нарушения когнитивных функций.

Контакты: larionova.ekaterin@gmail.com

Шувалова Алиса Александровна - клинический психолог, Московский научно-практический центр наркологии.

Сфера научных интересов: гашишизм как субкультура, последствия употребления каннабиноидов, психологические механизмы формирования зависимости, психотерапия зависимых и созависимых лиц, психотерапия детских неврозов.

Контакты: AlisaShuvalova@yandex.ru

\title{
A Review of Contemporary Research on the Influence of Tetrahydrocannabinol on Higher Mental Functions
}

\author{
Ekaterina V. Larionova ${ }^{\mathrm{a}, \mathrm{b}}$, Alisa A. Shuvalova ${ }^{\mathrm{b}}$ \\ ${ }^{a}$ Institute of Higher Nervous Activity and Neurophysiology of RAS, 5A Butlerova Str., Moscow, 117485, \\ Russian Federation \\ ${ }^{b}$ Moscow Research and Practical Centre for Narcology, $37 / 1$ Lyublinskaya Str., Moscow, 109390, Russian \\ Federation
}

\begin{abstract}
Currently cannabis is the most frequently used psychoactive substance, so the impact of cannabis on public health, particularly on the central nervous system, is a hot topic for research. This review aims to answer two questions: what are consequences of cannabis use and how stable are they? There are only a few studies of cannabis use in Russia. Foreign studies show mainly disorders of executive function and working memory in cannabinoid users. However, approaches used in foreign studies substantially differ from qualitative studies of higher mental functions which are specific to Russian neuropsychology. The majority of works are devoted to the study of the acute effects, and much less attention is given to long-term effects of cannabis. But growing evidence suggests that deficits of higher mental functions persist at least a month after the last dose of the drug after cannabis use is discontinued. In our opinion the use of the syndromic analysis through qualitative and quantitative studies would be effective for the investigation of possible disorders of higher mental functions in cannabinoid users and would answer the question of how stable these disorders are.
\end{abstract}

Keywords: higher mental functions, cannabinoids, executive functions, memory, THC.

\section{References}

Barnett, G., Licko, V., \& Thompson, T. (1985). Behavioral pharmacokinetics of marijuana. Psychopharmacology, 1, 51-56. 
Battistella, G., Fornari, E., Thomas, A., Mall, J. F., Chtioui, H., Appenzeller, M., ... Giroud, C. (2013). Weed or wheel! FMRI, behavioural, and toxicological investigations of how cannabis smoking affects skills necessary for driving. PLoS One, 8(1), 525-545. doi:10.1371/journal.pone.0052545

Böcker, K. B., Hunault, C. C., Gerritsen, J., Kruidenier, M., Mensinga, T. T., \& Kenemans, J. L. (2010). Cannabinoid modulations of resting state EEG power and working memory are correlated in humans. Journal of Cognitive Neuroscience, 22(9), 1906-1916. doi:10.1162/jocn.2009.21355

Bolla, K. I., Brown, K., Eldreth, D., Tate, K., \& Cadet, J. L. (2002). Dose-related neurocognitive effects of marijuana use. Neurology, 9, 1337-1343.

Bosker, W. M., Karschner, E. L., Lee, D., Goodwin, R. S., Hirvonen, J., \& Innis, R. B. (2013). Psychomotor function in chronic daily Cannabis smokers during sustained abstinence. PLoS One, 8, e53127. doi:10.1371/journal.pone.0053127

Bossong, M. G., Jansma, J. M., van Hell, H. H., Jager, G., Oudman, E., Saliasi, E., ... Ramsey, N. F. (2012). Effects of 9-tetrahydrocannabinol on human working memory function. Biological Psychiatry, 71(8), 693-699. doi:10.1016/j.biopsych.2012.01.008

Broyd, S. J., van Hell, H. H., Beale, C., Yücel, M., \& Solowij, N. (2016). Acute and chronic effects of cannabinoids on human cognition - A systematic review. Biological Psychiatry, 79(7), 557-67. doi:10.1016/j.biopsych.2015.12.002

Bryun, E. A., Shuvalova, A. A., \& Tsvetkov, A. V. (2014). Features of higher mental functions of people taking cannabinoids. Theory and Practice of Social Development, 19. Retrieved from http://teoriapractica.ru/rus/files/arhiv_zhurnala/2014/19/psychology/bryun-tsvetkov-shuvalova.pdf (in Russian)

Day, A. M., Metrik, J., Spillane, N. S., \& Kahler, C. W. (2013). Working memory and impulsivity predict marijuana-related problems among frequent users. Drug and Alcohol Dependence, 131(1-2), 171-174. doi:10.1016/j.drugalcdep.2012.12.016

Eggan, S. M., Melchitzky, D. S., Sesack, S. R., Fish, K. N., \& Lewis, D. A. (2010). Relationship of cannabinoid CB1 receptor and cholecystokinin immunoreactivity in monkey dorsolateral prefrontal cortex. Neuroscience, 169(4), 1651-1661.

Eldreth, D. A., Matochik, J. A., Cadet, J. L., \& Bolla, K. I. (2004). Abnormal brain activity in prefrontal brain regions in abstinent marijuana users. NeuroImage, 23(3), 914-920.

Hart, C. L., \& van Gorp, W. (2001). Effects of acute smoked marijuana on complex cognitive performance. Neuropsychopharmacology, 5, 757-765.

Hell, H. H., Bossong, M. G., \& Jager, G. (2011). Evidence for involvement of the insula in the psychotropic effects of THC in humans: a double-blind, randomized pharmacological MRI study. International Joumal of Neuropsychopharmacology, 10, 1377-1388. doi:10.1017/ S1461145711000526

Hester, R., Lubman, D. I., \& Yücel, M. (2010). The role of executive control in human drug addiction. Current Topics in Behavioral Neurosciences, 3, 301-318. doi:10.1007/7854_2009_28

Hooper, S. R., Woolley, D., \& De Bellis, M. D. (2014). Intellectual, neurocognitive, and academic achievement in abstinent adolescents with cannabis use disorder. Psychopharmacology, 231, 1467-1477.

Jager, G., \& Kahn, R. S. (2006). Long-term effects of frequent cannabis use on working memory and attention: an fMRI study. Psychopharmacology, 3, 358-368.

Lichko, A. E. (1991). Podrostkovaya narkologiya: rukovodstvo dlya vrachei [Adolescent narcology: A handbook for doctors]. Leningrad: Meditsina.

Luria, A. R. (1962). Vysshie korkovye funktsii cheloveka i ikh narushenie pri lokal'nykh porazheniyakh mozga [Human higher cortical functions and their impairment in local brain damage]. Moscow: Moscow University Press. 
Lyons, M. J., Bar, J. L., \& Panizzon, M. S. (2004). Neuropsychological consequences of regular marijuana use: a twin study. Psychological Medicine, 7, 1239-1250.

McDonald, J., Schleifer, L., Richards, J. B., \& de Wit, H. (2003). Effects of THC on behavioral measures of impulsivity in humans. Neuropsychopharmacology, 7, 1356-1365.

Medina, K. L., Hanson, K. L., Schweinsburf, A. D., Cohen-Zion, M., Nafel, B. J., \& Tapert, S. F. (2007). Neuropsychological functioning in adolescent marijuana users: Subtle deficits detectable after a month of abstinence. Journal of the International Neuropsychological Society, 13, 807-820.

Meier, M. H., Caspi, A., Ambler, A., Harrington, H., Houts, R., \& Keefe, R. S. (2012). Persistent cannabis users show neuropsychological decline from childhood to midlife. Proceedings of the National Academy of Sciences of the United States of America, 109, E2657-2664.

Pope, H. G. Jr., Gruber, A. J., Hudson, J. I., Huestis, M. A., \& Yurgelun-Todd, D. (2001). Neuropsychological performance in long-term cannabis users. Archives of General Psychiatry, 58(10), 909-915.

Pope, H. G. Jr., \& Yurgelun-Todd, D. (1996). The residual cognitive effects of heavy marijuana use in college students. The Journal of the American Medical Association, 275(7), 521-527. doi:10.1001/jama.1996.03530310027028

Ramaekers, J. G., Kauert, G., \& van Ruitenbeek, P. (2006). High-potency marijuana impairs executive function and inhibitory motor control. Neuropsychopharmacology, 10, 2296-2303.

Ranganathan, M., \& D'Souza, D. C. (2006). The acute effects of cannabinoids on memory in humans: a review. Psychopharmacology, 4, 425-444.

Shabanov, P. D. (2002). Osnovy narkologii [Fundamentals of narcology]. Saint Petersburg: Lan'.

Solowij, N., Stephens, R. S., \& Roffman, R. A. (2002). Cognitive functioning of long-term heavy cannabis users seeking treatment. The Journal of the American Medical Association, 9, 1123-1131.

Thames, A. D., Arbid, N., \& Sayegh, P. (2014). Cannabis use and neurocognitive functioning in a nonclinical sample of users. Addictive Behaviors, 39, 994-999.

Tsvetkov, A. V. (2012). The peculiarities of the mental activity of persons, addicted to cannabinoids. In V se"zd Obshcherossiiskoi obshchestvenno organizatsii "Rossiiskoe psikhologicheskoe obshchestvo". Moskva, 14-18 fevralya 2012 goda. Nauchnye materialy [The 5th Congress of the All-Russian nongovernmental organization "Russian Psychological Society", Moscow, 14-18 of February, 2012] (Vol. 3, pp. 72-73). Moscow: Rossiiskoe psikhologicheskoe obshchestvo. Retrieved from http://www.psyrus.ru/periodicals/pdf/v_tom_3.pdf (in Russian)

Ekaterina V. Larionova - junior research fellow, Institute of Higher Nervous Activity and Neurophysiology of RAS; clinical psychologist, Moscow Research and Practical Centre for Narcology.

E-mail: larionova.ekaterin@gmail.com

Alisa A. Shuvalova - clinical psychologist, Moscow Research and Practical Centre for Narcology. E-mail: AlisaShuvalova@yandex.ru 